\title{
BMJ Open Upper gastrointestinal bleeding as a risk factor for dialysis and all-cause mortality: a cohort study of chronic kidney disease patients in Taiwan
}

\author{
Chih-Chia Liang, ${ }^{1,2,3,4}$ Che-Yi Chou, ${ }^{1,2}$ Chiz-Tzung Chang, ${ }^{1,2}$ I-Kuan Wang, ${ }^{1,2}$ \\ Chiu-Ching Huang ${ }^{1,2,3}$
}

To cite: Liang C-C, Chou C-Y, Chang C-T, et al. Upper gastrointestinal bleeding as a risk factor for dialysis and all-cause mortality: a cohort study of chronic kidney disease patients in Taiwan. BMJ Open 2016;6:e010439. doi:10.1136/bmjopen-2015010439

- Prepublication history for this paper is available online. To view these files please visit the journal online (http://dx.doi.org/10.1136/ bmjopen-2015-010439).

$\mathrm{C}-\mathrm{CL}$ and $\mathrm{C}-\mathrm{YC}$ contributed equally.

Received 3 November 2015 Revised 13 April 2016 Accepted 14 April 2016

CrossMark

For numbered affiliations see end of article.

Correspondence to Professor Chiu-Ching Huang; cch@mail.cmuh.org.tw

\section{ABSTRACT}

Objective: Impaired renal function is associated with higher risk of upper gastrointestinal bleeding (UGIB) in patients with chronic kidney disease and not on dialysis (CKD-ND). It is unclear if UGIB increases risk of chronic dialysis. The aim of the study was to investigate risk of chronic dialysis in CKD-ND patients with UGIB.

Setting: All CKD-ND stage 3-5 patients of a CKD programme in one hospital between 2003 and 2009 were enrolled and prospectively followed until September 2012.

\section{Primary and secondary outcome measures:}

Chronic dialysis (dialysis for more than 3 months) started and all-cause mortality. The risk of chronic dialysis was analysed using Cox proportional hazard regression with adjustments for age, gender and renal function, followed by competing-risks analysis.

Results: We analysed 3126 CKD-ND patients with a mean age of $65 \pm 14$ years for 2.8 years. Of 3126 patients, 387 (12.4\%) patients developed UGIB, 989 (31.6\%) patients started chronic dialysis and 197 $(6.3 \%)$ patients died. UGIB increased all-cause mortality (adjusted HR (aHR): $1.51,95 \% \mathrm{Cl} 1.07$ to 2.13 ) and the risk of chronic dialysis (aHR; $1.29,95 \%$ $\mathrm{Cl} 1.11$ to 1.50). The subdistribution HR (SHR) of UGIB for chronic dialysis (competing event: all-cause mortality) was 1.37 (95\% Cl 1.15 to 1.64$)$ in competing-risks analysis with adjustments for age, renal function, gender, diabetes, haemoglobin, albumin and urine protein/creatinine ratio.

Conclusions: UGIB is associated with increased risk of chronic dialysis and all-cause mortality in patients with CKD-ND stages $3-5$. This association is independent of age, gender, basal renal function, haemoglobin, albumin and urine protein levels.

\section{INTRODUCTION}

Upper gastrointestinal bleeding (UGIB) is a common gastrointestinal disorder in patients with chronic kidney disease and not receiving dialysis (CKD-ND). ${ }^{1}{ }^{2}$ In patients with

\section{Strengths and limitations of this study}

- Upper gastrointestinal bleeding not only increases all-cause mortality but also increases the risk of chronic dialysis in patients with chronic kidney disease (CKD).

- This association is shown in a cohort of patients with CKD with adjustments for confounders

- The generalisability of this finding may be limited by the study being conducted in one hospital and on a single ethnic group.

CKD-ND, the risk of UGIB is negatively associated with their renal function. ${ }^{1}$ UGIB not only increases the risk of hospitalisation but also increases in-hospital mortality in patients with CKD-ND and CKD 5 patients on dialysis. ${ }^{3}$ The increased all-cause mortality associated with UGIB is usually explained by the worsened comorbid conditions caused by the UGIB. $^{3-5}$

Dialysis may be needed in patients with CKD-ND when UGIB leads to decreased intravascular volume and subsequent prerenal acute kidney injury. ${ }^{6}$ Furthermore, incidence of UGIB episodes may be the deciding factor for progression of CKD and may lead to chronic dialysis if patients with CKD-ND do not recover from the acute kidney injury. However, the long-term effect of UGIB on the risk of chronic dialysis in patients with CKD-ND is not clear. The aim of the study was to investigate the risk of chronic dialysis after UGIB in patients with CKD-ND.

\section{MATERIALS AND METHODS}

Patient enrolment

All patients in the outpatient-based CKD programme of China Medical University Hospital (CMUH) from June 2003 to December 2009 were included. The CKD programme included 
patients with CKD-ND stages 3-5 diagnosed by nephrologists and no exclusion criteria were applied. The CKD programme, supported by National Health Insurance Taiwan, includes clinical endocrinologists, nephrologists, CKD nurses, dieticians and social workers. The purpose of the CKD programme is to monitor the progression of patients with CKD. Laboratory measurements and CKD education are performed at least four times a year. The UGIB was identified from review of medical records and the date of UGIB was recorded. All patients were followed to the date of initiation of chronic dialysis including haemodialysis and peritoneal dialysis, loss to follow-up, death or until September 2012. Two patients, who received kidney transplants, were excluded in the analysis because the timing of kidney transplantation was not related to uraemic symptoms. As we aimed to investigate risk of chronic dialysis, patients who received dialysis for less than 3 months and patients who discontinued dialysis because of a recovery of renal function were excluded.

\section{Definition and measurements}

UGIB was defined as melena, haematemesis, or the presence of red blood in gastric lavage fluid, together with observation of a bleeding lesion or a lesion likely to have bled on endoscopy of the upper digestive tract. ${ }^{1}$ Coronary artery disease (CAD) was defined as a positive exercise test, angiographic findings of at least one stenosis of more than $50 \%$ or positive findings on scintigraphy. ${ }^{7}$ Diabetes mellitus was defined as use of insulin, use of oral antidiabetic medication or a fasting plasma glucose level of $126 \mathrm{mg} / \mathrm{dL}$ or more. ${ }^{8}$ Hypertension was defined as taking antihypertensives without regard to the actual measurement of blood pressure, or having a systolic blood pressure reading greater than $140 \mathrm{~mm} \mathrm{Hg}$ or a diastolic blood pressure reading greater than $90 \mathrm{~mm} \mathrm{Hg}{ }^{9}$ Patients' primary kidney disease, including diabetes nephropathy, chronic glomerulonephritis (CGN) and hypertensive nephropathy, was diagnosed by the primary care physician and recorded at enrolment.

Each patient's body mass index (BMI), blood urea nitrogen (BUN) and creatinine were measured at enrolment. Serum calcium, phosphorus, albumin, urine albumin/creatinine ratio (ACR) and urine protein/creatinine ratio (PCR) were measured 3 months after enrolment. Estimated glomerular filtration rate (eGFR) was calculated using the Modification of Diet in Renal Disease (MDRD) Collaboration formula. ${ }^{10}$ The formula for white or other race was used because the patients were all Chinese. The time-varying analysis used an average of three eGFR measurements. Medications associated with a higher risk of UGIB, including steroids, non-steroidal anti-inflammatory drugs (NSAIDs), aspirin, clopidogrel and warfarin, were recorded based on the review of medical records.

\section{Outcomes measurements}

The CKD nurses recorded the outcomes (dialysis for more than 3 months or mortality) and date of the outcomes in the computerised case-management system. The CKD nurses also checked patients' status in the computerised medical record system in CMUH and contacted patients who did not return to the outpatient every 6 months. The percentage of patients who were lost to follow-up was $6 \%$ in the CKD programme. The outcomes were validated by a study nurse through a review of medical records and the study nurse was blinded to the aim of the study, and physicians performed the analysis.

\section{Statistical analysis}

Data were reported as mean $\pm \mathrm{SD}$, median (IQR), or frequency (percentage), as appropriate. All continuous variables were tested using skewedness and kurtosis test for their normality. Data were analysed using Student's t test for parametric variables, Kolmogorov-Smirnov test for non-parametric variables, or $\chi^{2}$ test for categorical variables. Factors with a p value $<0.05$ in univariable Cox proportional hazard regression and risk factors of chronic dialysis in the literature were considered as possible confounders. Possible confounders for all-cause mortality and chronic dialysis were analysed using multivariable Cox proportional hazard regression. An adjusted HR (aHR) and 95\% CIs of aHR in multivariable Cox regression with adjustments for age, gender and eGFR were calculated. We utilised competing-risks analysis ${ }^{11}$ with adjustments for confounders identified in Cox regression because the risk of chronic dialysis may be overestimated if patients' death was not taken into consideration. ${ }^{12}{ }^{13}$ An adjusted subdistribution HR (aSHR) and $95 \%$ CI of aSHR were calculated in competing-risks analysis with adjustments for age, gender, eGFR and confounders for chronic dialysis. The primary outcome was defined as chronic dialysis and the competing event was patient's death in the competing-risks analysis. All analyses were performed using Stata V.13 SE (StataCorp, Texas, USA) and a $p$ value $<0.05$ was considered statistically significant.

\section{Ethical considerations}

Internal review board approval (DMR 99-IRB-301) was obtained for the review of medical records, and the need for informed consent was waived.

\section{RESULTS}

\section{Patient characteristics}

We identified 3126 patients with CKD-ND stages 3-5 including 1691 males and 1435 females, aged 65 \pm 14 years (table 1 ). Diabetes nephropathy and chronic glomerulonephritis (CGN) were the major forms of primary kidney disease. The basal eGFR of the patients was $21(11-37) \mathrm{mL} / \mathrm{min} / 1.73 \mathrm{~m}^{2}$ and the urine PCR was $1.4(0.5-3.6) \mathrm{g} / \mathrm{g}$ at enrolment. Patients with UGIB were older and more often had diabetes, a lower eGFR, lower haemoglobin, higher BUN, higher creatinine and lower serum albumin. They, however, had similar use of 
Table 1 Clinical characteristics and outcomes according to upper gastrointestinal bleeding

\begin{tabular}{|c|c|c|c|c|c|c|c|}
\hline \multirow{2}{*}{$\begin{array}{l}\text { Basal characteristics } \\
\text { Follow-up (year) }\end{array}$} & \multicolumn{2}{|c|}{$\begin{array}{l}\text { Total } \\
n=3126\end{array}$} & \multicolumn{2}{|c|}{$\begin{array}{l}\text { UGIB } \\
(-) n=2739\end{array}$} & \multicolumn{2}{|c|}{$\begin{array}{l}\text { UGIB } \\
(+) n=387\end{array}$} & \multirow{2}{*}{$\frac{\text { p Value }}{0.38}$} \\
\hline & 2.8 & $1.0-4.3$ & 2.8 & $0.8-4.3$ & 2.4 & $0.9-4.1$ & \\
\hline Age (year) & 65 & \pm 14 & 64 & \pm 14 & 68 & \pm 12 & $<0.01$ \\
\hline Male n (\%) & 1691 & 54.1 & 1476 & 53.9 & 215 & 55.6 & 0.54 \\
\hline \multicolumn{8}{|l|}{ Primary kidney disease } \\
\hline Diabetes nephropathy & 1141 & 36.5 & 968 & 35.3 & 173 & 44.7 & $<0.01$ \\
\hline CGN & 1059 & 33.9 & 938 & 34.3 & 121 & 31.3 & \\
\hline Hypertensive nephropathy & 520 & 16.6 & 476 & 17.4 & 44 & 11.4 & \\
\hline CAD & 380 & 12.2 & 324 & 11.8 & 56 & 14.5 & 0.14 \\
\hline eGFR (mL/min/1.73 m²) & 21 & $11-37$ & 21 & $11-38$ & 16 & $9-28$ & $<0.01$ \\
\hline BMI $\left(\mathrm{kg} / \mathrm{m}^{2}\right)$ & 24.2 & \pm 4.1 & 24.2 & \pm 4.1 & 24.1 & \pm 3.9 & 0.51 \\
\hline Haemoglobin (g/dL) & 10.6 & \pm 2.4 & 10.7 & \pm 2.4 & 10.1 & \pm 2.3 & $<0.01$ \\
\hline BUN (mg/dL) & 48 & \pm 31 & 48 & \pm 31 & 54 & \pm 31 & $<0.01$ \\
\hline Creatinine (mg/dL) & 3.8 & \pm 2.9 & 3.7 & \pm 2.8 & 4.3 & \pm 2.9 & $<0.01$ \\
\hline Calcium (mg/dL) & 9.1 & \pm 0.7 & 9.1 & \pm 0.7 & 9.1 & \pm 0.8 & 0.77 \\
\hline Phosphorus (mg/dL) & 4.6 & \pm 1.4 & 4.5 & \pm 1.4 & 4.6 & \pm 1.2 & 0.26 \\
\hline Albumin (g/dL) & 3.4 & \pm 0.6 & 3.5 & \pm 0.6 & 3.3 & \pm 0.6 & $<0.01$ \\
\hline ACR $(g / g)$ & 0.6 & $0.1-2.2$ & 0.5 & $0.1-2.0$ & 1.6 & $0.2-3.5$ & 0.08 \\
\hline PCR $(g / g)$ & 1.4 & $0.5-3.6$ & 1.3 & $0.5-3.4$ & 2.2 & $0.9-4.5$ & $<0.01$ \\
\hline \multicolumn{8}{|l|}{ Medications n (\%) } \\
\hline Steroids & 106 & 3.4 & 97 & 3.5 & 9 & 2.3 & 0.21 \\
\hline NSAIDs & 61 & 2.0 & 50 & 1.8 & 11 & 2.8 & 0.18 \\
\hline Aspirin & 351 & 11.2 & 305 & 11.1 & 46 & 11.9 & 0.66 \\
\hline Clopidogrel & 94 & 3.0 & 83 & 3.0 & 11 & 2.8 & 0.84 \\
\hline Warfarin & 64 & 2.1 & 52 & 1.9 & 12 & 3.1 & 0.12 \\
\hline
\end{tabular}

steroid, NSAIDs, aspirin, clopidogrel and warfarin. There were no differences in serum calcium and phosphate levels of the patients with UGIB and the patients without. The times of follow-up were similar in the patients with UGIB and the patients without $(\mathrm{p}=0.38$, table 2). The proportion of the patients with UGIB who started chronic dialysis was higher than that of the patients without UGIB $(61.5 \%$ vs $27.4 \%, \mathrm{p}<0.01)$. Patients with UGIB were associated with a higher allcause mortality than patients without UGIB (11.6\% vs $5.6 \%, \mathrm{p}<0.01)$.

\section{UGIB and risk of mortality}

UGIB was independently associated with higher all-cause mortality (table 3) with an aHR of 1.51 (95\% CI 1.07 to 2.13, $\mathrm{p}=0.02$ ). Patients' age, eGFR and serum albumin were independently associated with all-cause mortality.
The aHR was 1.05 for every one additional year (95\% CI 1.04 to $1.07, \mathrm{p}<0.01), 0.99$ for every $\mathrm{mL} / \mathrm{min} / 1.73 \mathrm{~m}^{2}$ of eGFR (95\% CI 0.0 .98 to $1.00, \mathrm{p}<0.01$ ) and 0.52 per $\mathrm{g} /$ $\mathrm{dL}$ of serum albumin (95\% CI 0.42 to $0.65, \mathrm{p}<0.01)$. Diabetes, CAD, haemoglobin and PCR were not associated with all-cause mortality. Use of steroid, NSAIDs, clopidogrel and warfarin was not associated all-cause mortality, either.

\section{UGIB and risk of chronic dialysis}

UGIB was associated with an increased risk of chronic dialysis with an aHR of 1.29 (95\% CI 1.11 to 1.50 , $\mathrm{p}<0.01)$. Diabetes was independently associated with increased risk of chronic dialysis with an HR of 1.49 (95\% CI 1.30 to $1.72, \mathrm{p}<0.01)$. Haemoglobin and serum albumin were associated with lower risk of chronic dialysis. PCR was associated with increased risk of chronic

Table 2 Outcomes of patients with and without upper gastrointestinal bleeding

\begin{tabular}{|c|c|c|c|c|c|c|c|}
\hline \multirow{2}{*}{$\begin{array}{l}\text { Outcomes } \\
\text { Follow-up (year) }\end{array}$} & \multicolumn{2}{|c|}{$\begin{array}{l}\text { Total } \\
n=3126\end{array}$} & \multicolumn{2}{|c|}{$\begin{array}{l}\text { UGIB (-) } \\
\mathrm{n}=2739\end{array}$} & \multicolumn{2}{|c|}{$\begin{array}{l}\text { UGIB (+) } \\
n=387\end{array}$} & \multirow{2}{*}{$\frac{\text { p Value }}{0.38}$} \\
\hline & 2.8 & $1.0-4.3$ & 2.8 & $0.8-4.3$ & 2.4 & $0.9-4.1$ & \\
\hline Chronic dialysis $\mathrm{n}(\%)$ & 989 & 31.6 & 751 & 27.4 & 238 & 61.5 & $<0.01$ \\
\hline Mortality n (\%) & 197 & 6.3 & 152 & 5.6 & 45 & 11.6 & $<0.01$ \\
\hline
\end{tabular}


Table 3 Risk of all-cause mortality and chronic dialysis in patients with chronic kidney disease and upper gastrointestinal bleeding

\begin{tabular}{|c|c|c|c|c|c|c|}
\hline \multirow{3}{*}{$\frac{\text { Confounders }}{U^{\text {UGIB }}}$} & \multicolumn{3}{|c|}{ All-cause mortality } & \multicolumn{3}{|c|}{ Chronic dialysis } \\
\hline & \multirow{2}{*}{$\begin{array}{l}\text { aHR } \\
1.51\end{array}$} & \multicolumn{2}{|c|}{$95 \% \mathrm{CI}$} & \multirow{2}{*}{$\begin{array}{l}\text { aHR } \\
1.29\end{array}$} & \multicolumn{2}{|c|}{$95 \% \mathrm{Cl}$} \\
\hline & & 1.07 & 2.13 & & 1.11 & 1.50 \\
\hline Age (per year) & 1.05 & 1.04 & 1.07 & 0.99 & 0.99 & 0.99 \\
\hline Diabetes & - & - & - & 1.49 & 1.30 & 1.70 \\
\hline eGFR $\left(\right.$ per $\left.\mathrm{mL} / \mathrm{min} / 1.73 \mathrm{~m}^{2}\right)$ & 0.99 & 0.98 & 1.00 & 0.94 & 0.93 & 0.94 \\
\hline Haemoglobin (per $\mathrm{g} / \mathrm{dL}$ ) & - & - & - & 0.74 & 0.66 & 0.82 \\
\hline Albumin (per $\mathrm{g} / \mathrm{dL}$ ) & 0.52 & 0.42 & 0.65 & 1.03 & 1.02 & 1.05 \\
\hline PCR (per $\mathrm{g} / \mathrm{g}$ ) & - & - & - & 1.29 & 1.11 & 1.50 \\
\hline
\end{tabular}

*Patients without UGIB as reference.

eGFR, estimated glomerular filtration rate using MDRD formula; PCR, urine protein/creatinine ratio; UGIB, upper gastrointestinal bleeding.

dialysis with an HR of 1.29 (95\% CI 1.11 to 1.50$)$ per $1 \mathrm{~g} / \mathrm{g}$. Steroids, NSAIDs, clopidogrel and warfarin were not associated with the risk of chronic dialysis. The aSHR of UGIB for chronic dialysis was 1.37 (95\% CI 1.15 to $1.64, \mathrm{p}<0.01)$ in competing-risks analysis with adjustments for age, gender, eGFR, diabetes, haemoglobin, albumin and PCR. The cumulative incidence of chronic dialysis in patients with UGIB and those without is shown in figure 1.

\section{DISCUSSION}

In this cohort of patients with CKD-ND stages 3-5, UGIB was associated with higher risk of chronic dialysis. The association of UGIB and risk of chronic dialysis was further validated using competing-risks analysis. The competing-risks analysis was critical to this study because UGIB was associated with increased all-cause mortality. UGIB can be a complication of uraemia because of the uraemic coagulopathy. In this study, patients with UGIB started chronic dialysis after a median of 1.2 (IQR: 0.7-

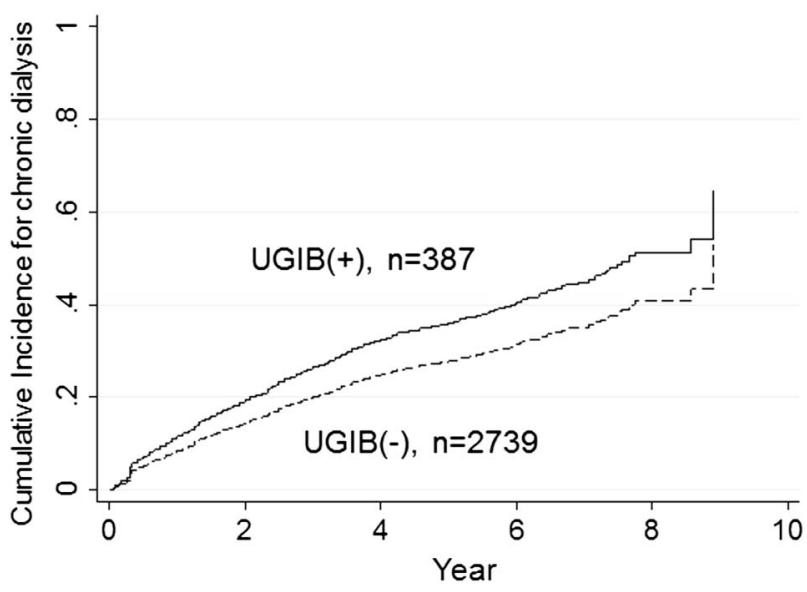

Figure 1 Cumulative incidence of chronic dialysis in patients with and without upper gastrointestinal bleeding (UGIB), using competing-risks regression with adjustments for age, gender, renal function, diabetes, haemoglobin, albumin and urine protein/creatinine ratio. The primary event was chronic dialysis and the competing event was all-cause mortality.
1.3) years. Uraemic coagulopathy associated UGIB therefore may not be one of the uraemic symptoms that required dialysis in these patients. In summary of our previous studies $^{114} 15$ and this study (figure 2), patients with CKD-ND were more at risk for developing UGIB, and UGIB may further accelerate the progression of CKD to chronic dialysis. The common aggravating factors of CKD and UGIB are anaemia, ${ }^{16}$ diabetes and hypoalbuminaemia. ${ }^{1}$ In addition, higher GFR and uses of ACE inhibitor (ACEi) or angiotensin II receptor blocker (ARB) may protect UGIB in this scenario. ${ }^{15}$ Some beneficial effects of ACEi and ARB may come from their association with decreased risk of UGIB. ${ }^{15}$

Most patients with CKD died from CAD during the course of CKD progression and only some of the patients with CKD started chronic dialysis. ${ }^{17-19}$ In our study, the proportion of patients who started chronic dialysis was higher than that of patients who died. This was not only observed in our previous studies ${ }^{120}$ but also in other cohorts of patients with CKD in Taiwan. ${ }^{21}$ The high prevalence of chronic dialysis in Taiwan may be explained by an ethnic difference ${ }^{22}$ or the effectiveness of multidisciplinary care for CKD in Taiwan. ${ }^{21}$

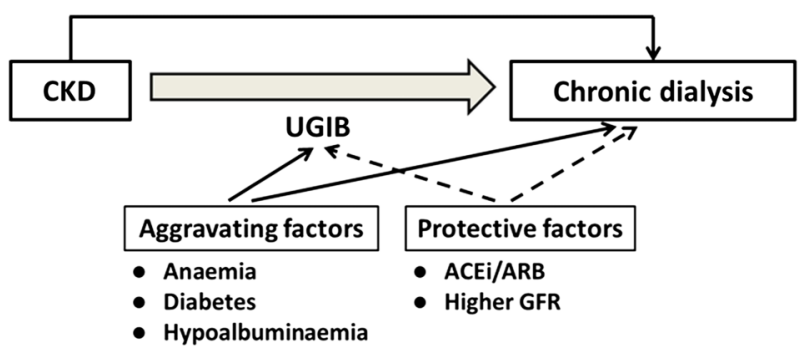

Figure 2 Upper gastrointestinal bleeding (UGIB) may accelerate the progression of chronic kidney disease (CKD) and further increase the risk of chronic dialysis. In this interactive scheme of UGIB and CKD, aggravating factors include anaemia, diabetes and hypoalbuminaemia, and protective factors contain a higher glomerular filtration rate (GFR) and include use of an ACE inhibitor (ACEi)/angiotensin II receptor blocker (ARB). 
Diabetes was associated with the risk of chronic dialysis because diabetes is a well-known confounder of dialysis. NSAIDs were also well-known risk factors for UGIB and chronic dialysis, ${ }^{23}$ but we did not find any significant association of NSAIDs and chronic dialysis. Aspirin, clopidogrel and warfarin were not associated with the risk of chronic dialysis in patients with CAD, either. Steroids treatment was relatively common in patients with $\mathrm{CKD}^{24}$ but only $3 \%$ of patients took steroids in this study. Steroids treatment was not associated with the risk of chronic dialysis in this study. The limited number of patients who took these medications in this study may explain this result. In addition, nausea and vomiting can be uraemic symptoms ${ }^{25}$ and are indications of dialysis. Nausea and vomiting are common in patients with UGIB. ${ }^{26}$ Although some patients with CKD initiated dialysis because of UGIB-related nausea and vomiting rather than uraemic symptoms, the percentages of patients with nausea and vomiting at the initiation of dialysis were similar among patients with UGIB and in those without. In addition, we excluded patients who underwent dialysis for less than 3 months and those who discontinued dialysis because of a recovery of renal function. UGIB-related nausea/vomiting did not explain the association of UGIB and chronic dialysis in this study.

The limitations of this study were its observational study design and the inclusion of all patients in a single hospital in Taiwan. This may limit the generalisability of our finding to other ethnic groups. More studies of various ethnic groups and multiple institutions are required to confirm our results. Patients of a CKD programme were analysed in this study and therefore patient selection bias cannot be avoided. We also recorded proton-pump inhibitor treatment for UGIB but did not find any beneficial effect of this treatment in decreasing risk of chronic dialysis and all-cause mortality. In spite of its limitations, this is the first study to show an association of UGIB and chronic dialysis, independent of all confounders of chronic dialysis. This suggests that UGIB not only leads to acute kidney injury but also results in long-term consequence of irreversible kidney injury in this population.

\section{CONCLUSIONS}

UGIB is associated with increased risk of chronic dialysis in patients with CKD stages 3-5 and not receiving dialysis. This association is independent of age, gender, basal renal function, diabetes, haemoglobin, albumin and urine protein levels. More studies will be warranted to investigate if prevention of UGIB may slow the progression of CKD and delay the need of dialysis in patients with CND-ND.

\section{Author affiliations}

${ }^{1}$ Kidney Institute and Division of Nephrology, Department of Internal Medicine, China Medical University Hospital, Taichung, Taiwan ${ }^{2}$ College of Medicine, China Medical University, Taichung, Taiwan
${ }^{3}$ Graduate Program of Translational Medicine, China Medical University, Taichung, Taiwan

${ }^{4}$ Institute of Biomedical Sciences, Academia Sinica, Taipei, Taiwan

Contributors C-CL was responsible for study concept, design and acquisition of data. C-YC was responsible for analysis, interpretation of data and drafting of the manuscript. C-TC and I-KW conducted critical revision of the manuscript for important intellectual content. C-CH was responsible for study supervision.

Funding This study is supported in part by the Taiwan Ministry of Health and Welfare Clinical Trial and Research Center of Excellence

(MOHW105-TDU-B-212-133019) and China Medical University Hospital (DMR-101-019 and DMR-102-015). The funders had no role in study design, data collection and analysis, decision to publish, or preparation of the manuscript.

Competing interests None declared.

Ethics approval China Medical University \& Hospital Research Ethics Committee.

Provenance and peer review Not commissioned; externally peer reviewed.

Data sharing statement No additional data are available.

Open Access This is an Open Access article distributed in accordance with the Creative Commons Attribution Non Commercial (CC BY-NC 4.0) license, which permits others to distribute, remix, adapt, build upon this work noncommercially, and license their derivative works on different terms, provided the original work is properly cited and the use is non-commercial. See: http:// creativecommons.org/licenses/by-nc/4.0/

\section{REFERENCES}

1. Liang CC, Wang SM, Kuo HL, et al. Upper gastrointestinal bleeding in patients with CKD. Clin J Am Soc Nephrol 2014;9:1354-9.

2. Yang JY, Lee TC, Montez-Rath ME, et al. Trends in acute nonvariceal upper gastrointestinal bleeding in dialysis patients. J Am Soc Nephrol 2012;23:495-506.

3. Sood P, Kumar G, Nanchal R, et al. Chronic kidney disease and end-stage renal disease predict higher risk of mortality in patients with primary upper gastrointestinal bleeding. Am J Nephrol 2012;35:216-24.

4. Åhsberg $\mathrm{K}$, Höglund $\mathrm{P}$, Staël von Holstein $\mathrm{C}$. Mortality from peptic ulcer bleeding: the impact of comorbidity and the use of drugs that promote bleeding. Aliment Pharmacol Ther 2010;32:801-10.

5. Leontiadis GI, Molloy-Bland M, Moayyedi P, et al. Effect of comorbidity on mortality in patients with peptic ulcer bleeding: systematic review and meta-analysis. Am J Gastroenterol 2013;108:331-45; quiz 46.

6. Moreau R, Lebrec D. Acute renal failure in patients with cirrhosis: perspectives in the age of MELD. Hepatology 2003;37:233-43.

7. Dormandy JA, Charbonnel B, Eckland DJ, et al. Secondary prevention of macrovascular events in patients with type 2 diabetes in the PROactive Study (PROspective pioglitAzone Clinical Trial In macroVascular Events): a randomised controlled trial. Lancet 2005;366:1279-89.

8. Gottlieb DJ, Punjabi NM, Newman AB, et al. Association of sleep time with diabetes mellitus and impaired glucose tolerance. Arch Intern Med 2005;165:863-7.

9. Lavie P, Herer P, Hoffstein V. Obstructive sleep apnoea syndrome as a risk factor for hypertension: population study. BMJ 2000;320:479-82

10. Levey AS, Bosch JP, Lewis JB, et al. A more accurate method to estimate glomerular filtration rate from serum creatinine: a new prediction equation. Modification of Diet in Renal Disease Study Group. Ann Intern Med 1999;130:461-70.

11. Fine JP, Gray RJ. A proportional hazards model for the subdistribution of a competing risk. J Am Stat Assoc 1999;94:496-509.

12. Evans DW, Ryckelynck JP, Fabre E, et al. Peritonitis-free survival in peritoneal dialysis: an update taking competing risks into account. Nephrol Dialy Transplant 2010;25:2315-22.

13. Bechade C, Guittet L, Evans D, et al. Early failure in patients starting peritoneal dialysis: a competing risks approach. Nephrol Dialy Transplant 2014;29:2127-35. 
14. Liang $\mathrm{CC}$, Muo $\mathrm{CH}$, Wang IK, et al. Peptic ulcer disease risk in chronic kidney disease: ten-year incidence, ulcer location, and ulcerogenic effect of medications. PLOS ONE 2014;9:e87952.

15. Chou CY, Wang SM, Chang PH, et al. Angiotensin II receptor blocker prevents upper gastrointestinal bleeding in hypertensive patients with chronic kidney disease not on dialysis. Int J Clin Pract 2015;69:722-8.

16. Hsu TW, Liu JS, Hung SC, et al. Renoprotective effect of renin-angiotensin-aldosterone system blockade in patients with predialysis advanced chronic kidney disease, hypertension, and anemia. JAMA Intern Med 2014;174:347-54.

17. Winkelmayer WC, Levin R, Avorn J. Chronic kidney disease as a risk factor for bleeding complications after coronary artery bypass surgery. Am J Kidney Dis 2003;41:84-9.

18. Go AS, Chertow GM, Fan D, et al. Chronic kidney disease and the risks of death, cardiovascular events, and hospitalization. $N$ Engl Med 2004;351:1296-305.

19. Tangri N, Stevens LA, Griffith J, et al. A predictive model for progression of chronic kidney disease to kidney failure. JAMA 2011;305:1553-9.

20. Chen IR, Wang SM, Liang CC, et al. Association of walking with survival and RRT among patients with CKD stages 3-5. Clin J Am Soc Nephrol 2014;9:1183-9.
21. Wang SM, Hsiao LC, Ting IW, et al. Multidisciplinary care in patients with chronic kidney disease: a systematic review and meta-analysis. Eur J Intern Med 2015;26:640-5.

22. Xu R, Zhang L, Zhang $\mathrm{P}$, et al. Comparison of the prevalence of chronic kidney disease among different ethnicities: Beijing CKD survey and American NHANES. Nephrol Dial Transplant 2009;24:1220-6.

23. Plantinga L, Grubbs V, Sarkar U, et al. Nonsteroidal anti-inflammatory drug use among persons with chronic kidney disease in the United States. Ann Fam Med 2011;9. 423-30.

24. Levin A, Bakris GL, Molitch $M$, et al. Prevalence of abnormal serum vitamin D, PTH, calcium, and phosphorus in patients with chronic kidney disease: results of the study to evaluate early kidney disease. Kidney Int 2007;71:31-8.

25. Murtagh FE, Addington-Hall JM, Edmonds PM, et al. Symptoms in advanced renal disease: a cross-sectional survey of symptom prevalence in stage 5 chronic kidney disease managed without dialysis. J Palliat Med 2007;10:1266-76.

26. Roderick PJ, Wilkes HC, Meade TW. The gastrointestinal toxicity of aspirin: an overview of randomised controlled trials. $\mathrm{Br} \mathrm{J} \mathrm{Clin}$ Pharmacol 1993;35:219-26. 